\title{
Mediterranean diet as a lifestyle and dynamic food pattern
}

\author{
Ramón Estruch ${ }^{1,2,3}$ - Anna Bach-Faig ${ }^{1,4,5}$ \\ Keywords Mediterranean diet $\cdot$ food pattern $\cdot$ lifestyle $\cdot$ health
}

Published online: 28 November 2018

C) Springer Nature Limited 2018

This Supplement of the European Journal of Clinical Nutrition includes the proceedings of conferences presented at the XI International Mediterranean Diet Conference entitled Mediterranean Diet as a Lifestyle and Dynamic Food Pattern, which was held in Barcelona on April 2016 in the framework of the International Food and Beverage Exhibition, Alimentaria 2016 [1]. This year was the 20th anniversary of the Mediterranean Diet Foundation (MDF), the institution that has been devoted to spreading the advances of this food pattern and way of life since 1996, date in which the first International Congress on the Mediterranean Diet was held and the Barcelona Declaration on the Mediterranean Diet enacted. The MDF has been involved in the definition of the Mediterranean diet and its representation as a pyramid [2]. Among its tasks, the MDF has endeavored to collect scientific evidence and spread the solid evidence acquired in multiple studies on the benefits of the Mediterranean diet on many health outcomes. This includes results from landmark clinical studies such as the PREDIMED trial, which demonstrated that adherence to a traditional Mediterranean-type diet reduces the incidence of cardiovascular disease by about $30 \%$, the incidence of type2 diabetes mellitus by $40 \%$, and the incidence of metabolic

Anna Bach-Faig

abachf@uoc.edu

1 Mediterranean Diet Foundation, Barcelona, Spain

2 Department of Internal Medicine, Hospital Clinic, August Pi Sunyer Biomedical Research Institute (IDIBAPS), Barcelona, Spain

3 CIBEROBN (Center for Research on Obesity and Nutrition), Instituto de Salud Carlos III, Madrid, Spain

4 Faculty of Health Sciences, Universitat Oberta de Catalunya, Barcelona, Spain

5 Food and Nutrition Area, Barcelona Official College of Pharmacists, Barcelona, Spain syndrome by about $10 \%$ [3]. In addition, the MDF also collaborated in the international recognition of the Mediterranean Diet by UNESCO as an Intangible Cultural Heritage of Humanity [4], increasing the visibility of the benefits of Mediterranean Diet on health. The last achievement of the Mediterranean Diet has been its recognition as a healthy dietary pattern in the Dietary Guidelines for Americans 2015-2020 of the United States Department of Agriculture [5] and in several clinical guides elaborated by the major American and European scientific societies, such as the AHA (American Heart Association) [6], ASA (American Stroke Association) [7], and EHS (European Heart Association), among others.

In this past edition of the Mediterranean Diet Conference held in Barcelona (Spain), scientists from around the world corroborated its benefits and effectiveness on longevity, quality of life, and prevention of several chronic diseases. Moreover, it was stated that the Mediterranean diet should be understood more as a way of life than a mere dietary pattern, as the Greek word "diata" reflects. Thus, the topics that were covered, examined, and discussed in this Conference are summarized in the present supplement and cover a vast variety of disciplines and expertise, as described below.

With the purpose of expanding the knowledge on the health effects of the Mediterranean food components, some reviews are included such as "Extra virgin olive oil: more than a healthy fat" by Jose López Miranda from University of Cordoba (Spain) and "New evidence on the effect of wine on health" by Iris Shai from Ben-Gurion University of Negev (Israel). Meat consumption, in lesser frequency and amounts than vegetable foods, is also part of the traditional Mediterranean diet. After the WHO declarations on meat consumption recommendations, it was necessary to analyze the role of meat in a healthy diet. Thus, José Peñalvo from the Tufts University in Boston (USA) clarified this issue in a paper on "Mediterranean diet and cardio-metabolic health: what is the role of meat?". Factors that affect carotenoid 
level in human plasma is another aspect that is analyzed by Rosa M Lamuela-Raventós from the University of Barcelona (Spain), who compared the differences between Mediterranean and Northern population.

As stated, the Mediterranean diet should be considered a lifestyle, and to further explore this notion, an analysis of the importance of sleep patterns in obesity and overall health, the effects of happiness and other psychological aspects on longevity, and the relationship between physical activity, exercise, and health was included in the discussion. Marie Pierre St. Onge from the New York Obesity Research Center (USA) focused on "Sleep and meal timing influence food intake and its hormonal regulation in healthy adults," and $\mathrm{M}^{\mathrm{a}}$ Antonia Lizárraga from University of Barcelona (Spain) explored the relationship between physical activity, exercise, and health, taking about the concept of healthy lifestyle, beyond the diet.

Since the importance of gut microbiota on health is increasingly recognized, Francisco Tinahones from the University of Málaga (Spain) delivered a lecture on "Importance of gut microbiota in obesity."

Moving from science to society, the relationship between cities, neighborhoods, and food was discussed in the talk "Healthy urbanism: how the built environment determines our health and how to improve it." Manuel Franco from the University of Alcalá de Henares (Spain) highlighted the results of a study on Mediterranean urban food environments: The Heart Healthy Hoods study.

Since dietary patterns, including the Mediterranean diet, are changing over time, other dietary patterns as well as the updating of the Mediterranean diet were also analyzed. The traditional Mediterranean diet needs to be adapted to the modern era according to new lifestyles and new agriculture and environment constraints. Currently, there is an increasing trend to adopt other dietary options for health purposes, such as vegetarian, vegan, and raw diets. Joan Sabaté from Loma Linda University (USA) presented a "Narrative Review of the Diets of Two Cohorts of Vegetarians: Description, Health and Sustainability Outcomes." "New and traditional foods in a modernized Mediterranean diet model" is also covered in this supplement by Michel de Lorgeril from the University of Grenoble (France).

Finally, it would be useful to appraise adherence to the Mediterranean diet worldwide, since the results may be useful to implement agricultural and health policy actions to counteract dietary westernization and to prevent erosion of the healthy Mediterranean diet pattern due to the effects of socio-economic and lifestyle factors that threaten adherence to this dietary pattern and the landscapes, techniques, and knowledge related to it. As an example, Attilio Rigotti from Universidad Católica (Chile) explores the challenge of "promoting and implementing the Mediterranean Diet in the
Southern Hemisphere: the Chilean experience." The Mediterranean diet shares food components and culinary traditions with areas from the 40th parallel North and South such as California, China, South Africa, Chile, Argentina, and Australia.

The Parallel 40-World Mediterranean Diet Surveillance System (WMDSS) [8] has been designed by the MDF with the aim to collect, analyze, interpret, and disseminate information (particularly strategic indicators) on the Mediterranean diet to contribute to improve the knowledge, preservation, and expansion of this eating pattern and lifestyle on the Mediterranean area and also the worldwide areas located around the 40th parallel. In this framework, researchers from the MDF present an analysis on "Adherence to the Mediterranean Diet worldwide from the 60's to 2011 " to assess possible changes in adherence to this dietary pattern due to the last economic crisis by using the Food and Agriculture Organization Food Balance Sheets. Also, in order to develop tools for the promotion and prescription of the Mediterranean diet, investigators from the WMDSS covered the topic "Adherence of Spanish primary physicians and clinical practise to the Mediterranean Diet." Primary care physicians do not show a high level of adherence to the Mediterranean diet, which underlines the need to design adequate tools to evaluate adherence in their patients. This, together with the promotion of high-protein diets, also prescribed by doctors and specialists as a strategy for weight loss or maintenance, with a major impact on health, brings us to discuss nutrition knowledge and clinical practice in primary care settings.

We are confident that the contents of this special issue will contribute to deepen knowledge of current nutrition topics and illustrate scientific advances in order to provide to European citizens and key institutions the data and tools to safeguard the Mediterranean diet, a reference dietary pattern for community and clinical nutrition.

Funding This article is published as part of a supplement sponsored by the Mediterranean Diet Foundation and the Diputació de Barcelona. This publication is a part of the Paralelo40 - International Surveillance System on Mediterranean Diet.

\section{Compliance with ethical standards}

Conflict of interest R.E. has received lecture fees from Brewers of Europe, Belgium, Cerveceros de España, Spain, Fundación Dieta Mediterránea, Instituto Cervantes, Madrid, Interprofesional del Aceite de, Oliva, Madrid, Spain, Lilly Laboratories SA, Spain. R.E. has also received grant support from Consorcio Centro de Investigación Biomédica en Red (CIBER) Obesidad, Health Research Project grant from the Carlos III Institute of Health, Ministry of Economy and Competitiveness of Spain (FIS), La Marató Foundation of TV3, Grand Fontaine, SA, National Institute on Alcohol Abuse and Alcoholism (NIAAA), USA. The remaining authors declare that they have no conflict of interest. 


\section{References}

1. XI Mediterranean Diet Conference. XI International Mediterranean Diet Conference (27-28 of April (2016) in Barcelona). http:// congresodietamediterranea.com/en/. Accessed August 2017.

2. Bach-Faig A, Berry EM, Lairon D, Reguant J, Trichopoulou A, Dernini S, et al. Mediterranean diet pyramid today. Science and cultural updates. Public Health Nutr. 2011;14:2274-84.

3. Estruch R, Ros E, Salas-Salvadó J, Covas MI, Corella D, Arós F, et al. Primary prevention of cardiovascular disease with a Mediterranean diet. N Engl J Med. 2013;368:1279-90.

4. UNESCO (2010) Representative List of the Intangible Cultural Heritage of Humanity. https://ich.unesco.org/en/decisions/5.COM/ 6.41. Accessed August 2017.
5. Dietary Guidelines for Americans 2015-2020. Appendix 4. USDA Food Patterns: Healthy Mediterranean-Style Eating Pattern. https:// health.gov/dietaryguidelines/2015/guidelines/appendix-4/. Accessed August 2017.

6. American Heart Association. Mediterranean-style diet details. http://www.heart.org/HEARTORG/Affiliate/Mediterranean-stylediet-details_UCM_461758_Article.jsp\#.WY1rwdJJaM8. Accessed August 2017.

7. Kernan WN, Ovbiagele B, Black HR, Bravata DN, Chimowitz MI, Ezekowitz MD, et al. Guidelines for the prevention of stroke in patients with stroke and transient ischemic attack: a guideline for healthcare professionals from the American Heart Association/ American Stroke Association. Stroke. 2014;45:2160-236.

8. The Parallel 40-World Mediterranean Diet Surveillance System (WMDSS). http://www.paralelo40.es/en/. Accessed August 2017. 